\title{
Estilos de Aprendizagem e Rendimento Acadêmico na Universidade
}

\section{Learning Styles and Academic Performance at the University}

\author{
Alison Martins Meurer 1* \\ Daiana Rafaela Pedersini ${ }^{2}$ \\ Ricardo Adriano Antonelli ${ }^{2}$ \\ Simone Bernardes Voese ${ }^{1}$ \\ ${ }^{1}$ Universidade Federal do Paraná, Brasil \\ ${ }^{2}$ Universidade Tecnológica Federal do Paraná, Brasil
}

\begin{abstract}
A pesquisa teve como objetivo analisar se o rendimento acadêmico dos alunos do curso de Ciências Contábeis é distinto entre os estilos de aprendizagem e características observáveis nos discentes e docentes. Os dados foram coletados por meio da aplicação do Inventário de Estilos de Aprendizagem de Kolb (1984), em uma amostra com 94 alunos e 23 professores. Na análise dos dados foram utilizados testes de médias e regressão linear simples. Os resultados indicaram que $45,74 \%$ dos acadêmicos são do estilo convergente e $43,48 \%$ dos docentes são do estilo de aprendizagem assimilador. Dos vinte e seis agrupamentos analisados, sete apresentaram diferenças significativas no rendimento acadêmico, as quais são: reprovação, horas dedicadas semanalmente aos estudos extraclasse, nota de ingresso do ENEM, facilidade em Matemática e estilos coincidentes de aprendizagem docente com o discente. Os alunos que possuem o mesmo estilo de aprendizagem do professor tendem a ter um menor índice de reprovação, comprovando a teoria proposta por Kolb (1984). Com isto, a plataforma teórica abordada por Kolb (1984) auxilia a fomentar o debate e sustenta o desenvolvimento de estratégias para um ensino cíclico que atenda ao máximo as necessidades de aprendizagem existentes, em especial, para que a ação docente consiga atingir a todos os estilos de aprendizagem dos alunos.
\end{abstract}

Palavras-chave: Rendimento acadêmico; Aprendizagem; Universidades; Docente; Brasil.

\begin{abstract}
The research aimed at analyzing if the academic Accounting Sciences student's performance is different among the learning styles and observable characteristics academic and professors. Data were collected through the application of the Kolb Learning Styles Inventory (1984), in a sample with 94 students and 23 teachers. Linear regression and mean tests were used in the data analysis. The results indicated that $45.74 \%$ of the students are convergent style and $43.48 \%$ of the teachers are the assimilating learning style. Of the twenty-six groups analyzed, seven presented significant differences in academic performance, which are: reproof, dedicated weekly hours to extraclass studies, ENEM entrance note, ease in Mathematics and matching styles of teacher learning with the student. Students with the same teacher learning style tend to have a lower rate of reproof, proving the theory proposed by Kolb (1984). In this way, the theoretical platform addressed by Kolb (1984) helps to foment the debate and supports the strategies development for a cyclical education that meets the maximum of the existing learning needs, in particular, so that didactic teaching action can achieve all kinds of learning styles of students.
\end{abstract}

Keywords: Academic achievement; Learning; Universities; Teachers; Brazil.

*Contacto: alisonmmeurer@gmail.com

ISSN: 1696-4713

www.rinace.net/reice/

revistas.uam.es/reice
Recibido: $\quad 15$ de junio 2018

$1^{\text {a }}$ Evaluación: 21 de julio 2018

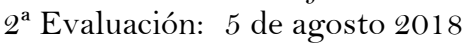

Aceptado: $\quad 26$ de agosto 2018 


\section{Introdução}

Durante as décadas de 1980, 1990 e 2000 os estudos com foco na aprendizagem cresceram significativamente. Souza e outros (2013) citam que diversos autores publicaram pesquisas e formularam teorias a respeito do assunto, colocando em foco as áreas da aprendizagem contínua e experiencial, com destaque para Kolb (1984), Kolb, Baker e Jensen (1997) e Larsen (2004).

Nesse contexto, Kolb (1984, p. 132) conceitua a aprendizagem como sendo um "processo por onde o conhecimento se desenvolve por meio da experiência”. A ocorrência de tal desenvolvimento difere entre os indivíduos, visto que cada ser possui um método e uma forma própria para desenvolver sua aprendizagem em que conhecer os diversos estilos de aprendizagem pode se tornar um diferencial na prática docente e na absorção do conhecimento (Albuquerque et al., 2015). Assim, o estilo de aprendizagem não é caracterizado pelo que a pessoa aprende, mas pelo comportamento manifestado para desenvolver o processo de aprendizagem (Butzke e Alberton, 2017). Adicionalmente, Correia e Moura Júnior (2017) indicam que os conceitos mais atuais de aprendizagem envolvem obter mais conhecimento, memorizar e reproduzir, aplicar fatos ou procedimentos, entender, ver algo de uma forma diferente ou transformar-se como pessoa.

Nesta linha, alguns estudos buscaram evidenciar os estilos de aprendizagem nas mais variadas áreas do conhecimento. $\mathrm{Na}$ área de negócios, especificamente em Ciências Contábeis e Administração, por Albuquerque e outros (2015) e Basílio e Vasconcellos (2011), respectivamente; nas ciências humanas, especificamente em Psicologia, Pimentel (2007) traz suas contribuições e, nas ciências exatas, no curso de Engenharia Civil, por Pereira (2005). Embora existam pesquisas relacionadas aos estilos de aprendizagem (Albuquerque et al., 2015; Basílio e Vasconcellos, 2011; Pereira, 2005; Pimentel, 2007), ainda há escassez de informações ligadas aos estilos de aprendizagem, características dos docentes e discentes e as possíveis diferenças no rendimento acadêmico dos alunos, a partir dessas diferentes características. Perante isso, é importante ressaltar que para o processo educacional é fundamental encontrar o melhor ou os melhores métodos para desenvolver o conhecimento, porém, para isso, anteriormente é necessário saber e conhecer o modo com que os discentes aprendem e os fatores que possam influenciar nessa aprendizagem, conforme destacado por Kuri (2004).

No que concerne à avaliação do processo de ensino-aprendizagem, o rendimento acadêmico vem sendo utilizado amplamente pelas Instituições de Ensino Superior (IES). Munhoz (2004) define rendimento acadêmico como uma forma de avaliação do aluno na execução de uma tarefa, essa atribuição avaliativa pode ser por meio de notas ou por conceitos obtidos. Normalmente, as IES utilizam algumas metodologias na mensuração do rendimento acadêmico, dentre elas destaca-se o Coeficiente de Rendimento Acadêmico (CRA). O CRA não possui uma fórmula específica, portanto pode se diferenciar entre IES, sendo realizado por meio de nota da disciplina, nota por período, média geral acumulada ou exames externos a IES (Miranda et al., 2015). Assim, esta variável apresenta-se como um dos principais indicadores do sucesso acadêmico, caracterizando como estudo relevante em conjunto com aspectos pedagógicos direcionados às formas de aprendizagem, além das características observáveis dos discentes, como por exemplo, horas de estudos, gênero e instituição onde cursou o Ensino Médio. 
Nesse sentido, Oliveira e outros (2013) destacam que os docentes precisam identificar as formas mais apropriadas de transmitir informações acerca do que é estudado em sala de aula, a fim de que os acadêmicos absorvam melhor o conteúdo e obtenham um desempenho acadêmico satisfatório. Desse modo, reconhecendo a relevância de abordar aspectos inerentes ao processo de ensino-aprendizagem, esta investigação é direcionada pela seguinte inquietação: o rendimento acadêmico dos alunos do curso de Ciências Contábeis é distinto entre os estilos de aprendizagem e características observáveis dos discentes e docentes? Tem-se como objetivo principal analisar se há diferenças estatisticamente significativas no rendimento acadêmico dos alunos do curso de Ciências Contábeis conforme os estilos de aprendizagem e as características observáveis dos discentes e docentes que lecionaram as disciplinas no decorrer do curso.

A presente pesquisa promove avanços nos achados ao realizar análises mais abrangentes, ao buscar identificar possíveis diferenças no rendimento acadêmico de acordo com fatores e características dos alunos e estilo de aprendizagem dos discentes e docentes. Nesse contexto, Oliveira e outros (2013) advertem que a adoção de um estilo de ensino diferente do estilo de aprendizagem, pode ser um dos motivos do baixo desempenho acadêmico por parte do aluno, sendo necessário observar os variados estilos de aprendizagem no planejamento de um curso, na disciplina ou em aula.

Lima e Silva (2013, p. 6) indicam "que o processo de aprendizagem se tornou mais complexo, o que tem ampliado a discussão sobre o papel do docente como um facilitador em sala de aula, incentivando os estudantes ao senso crítico, criativo e reflexivo". Assim, a análise conjunta docente e discente, ao se pesquisar aprendizagem é enaltecida, devido ao importante papel docente e à necessidade de atingir um maior número de alunos. Kuri (2004) aponta a existência de três fatores que influenciam na qualidade da aprendizagem dos estudantes: habilidade inata, formação e compatibilidade entre os estilos de aprendizagem e o ensino. Com relação aos dois primeiros fatores não há forma de controle por parte do professor, porém ambos fornecem possibilidades de autoconhecimento e de desenvolvimento humano e profissional, uma vez que auxilia os docentes no planejamento e na ação didático-pedagógica. A compatibilidade entre os estilos de aprendizagem e o de ensino diz respeito a observação das diferentes formas com que os alunos aprendem. Identificar os estilos de aprendizagem pode auxiliar na evidenciação de possíveis adaptações das metodologias de ensino utilizadas, contribuindo com a aprendizagem dos discentes do curso de Ciências Contábeis, preenchendo lacunas educacionais e melhorando o processo de aprendizagem.

\section{Fundamentação teórica}

Em seus estudos, Bordenave e Pereira (2011) citam que ensinar e aprender são ações distintas, pois um assunto ou teoria pode ser ensinada de maneira completa, sem que o conhecimento tenha sido assimilado ou compreendido pelos alunos. Na busca por ensinar de uma maneira que satisfaça a todos, Dib (1994) destaca que as críticas ao modelo tradicional de ensino crescem a cada dia devido a sua limitada adequação às necessidades dos alunos e da sociedade. Muitas vezes o processo de ensino é massificado, tratando a todos como iguais. Portanto, torna-se necessário que tal processo de transmissão de ensino/aprendizagem seja mais eficiente e eficaz, sendo destacada a relevância em considerar os estilos de aprendizagem no ambiente educacional. 
Para Oliveira e outros (2013) e Albuquerque e outros (2015) estilo de aprendizagem é o modo com que um indivíduo constrói e assimila o conhecimento, se relacionando com o seu comportamento durante o aprendizado e não necessariamente com o que ele consegue aprender. Por exemplo, algumas pessoas possuem facilidade para aprender a partir de conceitos teóricos, enquanto outros preferem a aplicação de situações reais. $\mathrm{Na}$ mesma linha de pensamento, Campbell, Campbell e Dickinson (2000, p. 161) afirmam que "os estilos de aprendizagem se referem às diferenças individuais na maneira como a informação é compreendida, processada e comunicada”. Os autores também citam que os educadores que respeitam as diferenças individuais entre os alunos, ao mesmo tempo em que apreciam e celebram a diversidade nas maneiras de aprender, ensinarão mais por meio de seus comportamentos do que por meio das estratégias.

Kuri (2004) ressalta que o melhor entendimento pelos educadores dos estilos de aprendizagem predominantes dos alunos é importante, pois pode despertar maior interesse discente ao conteúdo melhorando, inclusive, a comunicação entre educador/acadêmico. Assim, existem diversos instrumentos que visam à identificação do estilo de aprendizagem, entre os quais o Inventário de Estilo de Aprendizagem de Kolb tem maior aplicação e divulgação quando analisada a área Contábil (Honn e Ugrin, 2012). Esse instrumento se baseia no modelo teórico da aprendizagem experiencial desenvolvido por David Kolb buscando identificar os estilos de aprendizagem por meio de características comuns para avaliar e melhorar o método de ensino utilizado (Butzke e Alberton, 2017).

Para Kolb (1984), a aprendizagem divide-se em dois eixos, a percepção e o processamento. Cada eixo apresenta uma subdivisão em outras duas formas, conforme demonstrado no quadro 1.

Quadro 1. Percepções e processamentos

\begin{tabular}{l} 
Percepção \\
Experiência Concreta (EC): O indivíduo é aberto a novas situações e adapta-se às mudanças. \\
Concepção Abstrata (CA): Pessoas que procuram organizar intelectualmente as informações \\
por meio de conceitos, teorias e princípios que puderam captar. \\
\hline Processamento \\
Experiência Ativa (EA): O indivíduo se envolve e busca testar as situações para aprender. \\
Observação Reflexiva (OR): A pessoa busca observar o ambiente de diferentes maneiras, \\
confiando em seus pensamentos e sentimentos para formar opiniões sem precisar se envolver. \\
Fonte: Adaptado de Kolb (1984).
\end{tabular}

Por meio da combinação de uma percepção (EC ou CA) com um processamento (EA ou OR), surgem os quatro estilos (ou preferências) de aprendizagem. Os estilos de aprendizagem apresentados por Kolb (1984) são: acomodador, divergente, assimilador e convergente. $\mathrm{O}$ procedimento desenvolvido pelo autor consiste em identificar, por meio de um inventário com algumas afirmativas, as melhores formas com que a pessoa julga aprender. A partir desse resultado é possível descobrir seu estilo de aprendizagem e suas maneiras de assimilar o conhecimento.

Para Kuri (2004), o modelo de aprendizagem de Kolb é denominado experiencial por duas razões. A primeira é histórica, porque suas origens intelectuais estão apoiadas no trabalho de John Dewey, Kurt Lewin e Jean Piaget. A segunda razão é a ênfase atribuída ao papel da experiência no processo de aprendizagem, pois Kolb (1984) afirma que o conhecimento é criado por meio das experiências. 
O modelo de Kolb (1984) é pesquisado por diversos autores, avaliando os estilos de aprendizagem em vários cursos. Sobral (2005) afirma que a relevância do estudo de Kolb (1984) é notória não somente por ser um dos primeiros pesquisadores a formular um estudo e modelo completo, mas também por conseguir segregar em grupos e caracterizar os diferentes modos de aprender dos indivíduos.

Estudos como o de Basílio e Vasconcellos (2011) apontam que há correlação entre os estilos de aprendizagem dos alunos de Administração e o rendimento acadêmico. Leite Filho, Batista, Junior e Siqueira (2008) contrariando tal informação, apuraram que no curso de Ciências Contábeis não há correlação entre os estilos de aprendizagem e o rendimento acadêmico. Similarmente, o mesmo ocorre no curso de Medicina, resultado identificado por Pellón, Nome e Áran (2013).

Sonaglio, Godoi e Silva (2013) constataram que nos cursos de Bacharelado em Administração de uma universidade de Santa Catarina e outra da Paraíba, o estilo de aprendizagem com maior incidência é o assimilador, enquanto Souza e outros (2013) afirmam que no curso de Bacharelado em Administração da Universidade Federal de Alagoas, o estilo com predominância é o divergente. Similarmente, Turra, Jacomossi e Biavatt (2015), nos alunos do curso de Ciências Contábeis, o estilo predominante também é o divergente.

Porém, outra distinção é encontrada nas pesquisas de Albuquerque e outros (2015), Lima Filho, Bezerra e Silva (2016) e Santos, Bacinello e Hein (2017), que retratam a predominância de alunos no curso de Ciências Contábeis da Universidade Federal de Campina Grande com estilo de aprendizagem assimilador, contrariamente a Reis, Paton e Nogueira (2012), que apontam o estilo de aprendizagem convergente como o mais presente no curso de Ciências Contábeis de uma instituição pública e outra privada, ambas localizadas no estado do Paraná.

As disparidades entre alguns resultados são ancoradas no argumento exposto por Kolb (1984) de que o estilo de aprendizagem é modificado de acordo com as experiências. Tão logo, como os estudos citados foram elaborados em diferentes localidades, os ambientes e as experiências são dissonantes, justificando tais achados. Percebe-se que os estilos que mais se destacam na área de negócios são o assimilador e o convergente. Esses estilos estão diretamente ligados com a concepção abstrata, que utiliza o pensamento lógico como base fundamental à assimilação e ao desenvolvimento do conhecimento.

Freitas (2004) e Araújo e outros (2014) apontam que discentes do sexo feminino possuem melhor rendimento acadêmico. Quando verificada a modalidade de Ensino Médio cursada é percebido que os resultados são discordantes, Freitas (2004), Pedrossa e Tessler (2004) e Souza e outros (2013) afirmam que este fator não possui significância quanto ao rendimento acadêmico, apesar dos alunos vindos de escolas públicas possuírem maior desempenho. Já para Andrade (2007), os alunos de escolas particulares apresentam melhor coeficiente de rendimento acadêmico.

Ao analisar a idade dos estudantes, Freitas (2004) aponta que este fator não influencia no rendimento acadêmico. Entretanto, Araújo e outros (2014) asseguram que, quanto maior a idade, melhor tende a ser o rendimento. Já Miranda e outros (2013) apresentam que este fator é inconclusivo, ou seja, não se pôde verificar estatisticamente tal influência em seus resultados. 
Adicionalmente, algumas outras variáveis também já foram estudadas, como: (i) Oliveira e Santos (2005) verificaram que o tipo de avaliação e a interpretação de texto têm correlação com o rendimento acadêmico; (ii) Miranda e outros (2013) discorrem que a experiência do docente influencia no rendimento do discente; (iii) Silva (2013) constatou que quanto menor a nota no ENEM-Exame Nacional do Ensino Médio, menor tende a ser o rendimento no Ensino Superior; e (iv) para Nogueira e outros (2012) o rendimento acadêmico não é influenciado pelo estilo de aprendizagem.

Apesar de alguns estudos não terem apresentado fatores significantes quanto ao desempenho acadêmico, é possível elencar uma gama de variáveis que podem ter associação com o rendimento dos discentes, como elementos relacionados desde as características sociais e econômicas até a influência que os docentes ou os métodos educativos utilizados por eles durante suas aulas exercem sobre os alunos. Perante a diversas óticas que podem ser utilizadas para abordar o tema ressalta-se a importância dos estudos de Campbell (2011) e Silva, Oliveira e Rogers (2015), os quais analisaram as características psicológicas e comportamentos sociais que podem influenciar o rendimento acadêmico, contudo somente a variável do tempo dedicado ao estudo foi incorporada a esta pesquisa devido ao objetivo e ao norteamento proposto.

\section{Método}

A pesquisa se caracteriza em estudo descritivo, desenvolvido em um ambiente de campo que buscou captar a rotina real dos respondentes. Na presente pesquisa existem duas populações, a primeira compreendeu 152 alunos regularmente matriculados no curso de Ciências Contábeis de uma IES pública no ano de 2015, dentre os quais 108 responderam o instrumento de coleta, das quais 94 respostas foram consideradas válidas à análise. A segunda população refere-se aos docentes que lecionaram no ano de 2015 para a primeira amostra, que totalizou 23 professores, dos quais todos participaram do estudo, não tendo nenhum questionário invalidado.

Em face do enfoque do trabalho optou-se por realizar o estudo em somente uma instituição. A proposta de avaliar o estilo de aprendizagem docente-discente exigiu que fosse ponderada a exposição do aluno durante toda sua trajetória universitária com os diferentes estilos de aprendizagem dos professores de acordo com a carga horária das disciplinas e o tempo de atividade do professor, pois houve troca de discentes no decorrer do ano letivo. Desse modo, a análise da referida instituição mostra-se oportuna por mostrar de que forma o alinhamento dos estilos de aprendizagem do discente-docente pode afetar o desempenho do aluno, já que na literatura há indícios de que o professor tende a lecionar conforme ele julga aprender.

Foram aplicados dois tipos de questionários no levantamento dos dados, um para cada amostra da pesquisa, sendo que ambos eram compostos por dois blocos, com o "Bloco I. Dados dos Respondentes" que trata das características pessoais dos indivíduos, e o "Bloco II. Inventário de Kolb” teve como objetivo realizar a identificação dos estilos de aprendizagem por meio dos aspectos que o respondente mais valoriza no processo de aprendizagem.

Diante da utilização do Inventário de Kolb, é importante destacar que o instrumento já possui evidências de validade na realidade brasileira. Sobral (1992) indica que a análise da validade interna do inventário de Kolb, com relação a intercorrelação, consistência interna 
e estrutura de fatores das escalas, indica que a versão do instrumento conserva as características psicométricas do original. Posteriormente, Cerqueira (2000) contou com validação de definição com a colaboração de 30 juízes relatando que a concordância destes quanto à classificação do estilo de aprendizagem dos quatro blocos do instrumento de Kolb foi unânime.

Na sequência, quanto à estrutura do questionário, as questões $Q_{13}, Q_{14}$ e $Q_{15}$ utilizaram escala numérica de 11 níveis para verificar o nível de facilidade que os alunos auto atribuíam-se em Matemática, interpretação de texto e raciocínio lógico. No quadro 2 são demonstradas as perguntas do Bloco I dos questionários dos discentes e docentes, respectivamente.

Quadro 2. Bloco I. Dados do respondente

$Q_{01}$ Período predominante QQ 02 . Gênero

QQ ${ }_{\text {оз. Idade (em anos) }}$

\& QQ $Q_{04 .}$ Modalidade de Ensino Médio cursada

QQ $Q_{05}$. Modalidade de ingresso na instituição

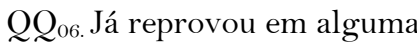
disciplina do curso de Bacharelado em Contábeis?

$\mathrm{QQ}_{07 .}$ Qual(is) foi(foram) a(s) disciplina(s)?

Qo1. Gênero

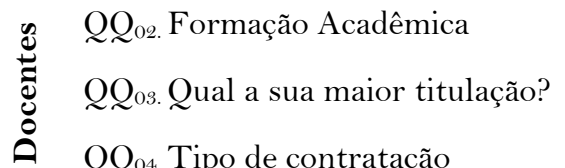
profissional

QQ ${ }_{05}$. Experiência docente

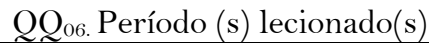

Q 08 . Qual a matéria preferida da grade curricular

Q 09 . Você possui outro curso superior?

$Q_{10}$. Em sua opinião qual é o melhor método de avaliar o conhecimento? (Somente uma alternativa).

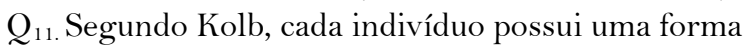
de aprender, assinale a alternativa que melhor define a sua forma de aprender. (Somente uma alternativa).

$Q_{12 .}$. Quanto tempo semanalmente você dedica aos estudos além das horas aulas?

$Q_{13 .}$ Facilidade em Matemática

$Q_{14}$. Facilidade em interpretação de texto $\mathrm{QQ}_{15}$. Facilidade em raciocínio lógico

QQ $_{07}$. Preencha os check-box conforme a quantidade de horas-aula que você lecionava por semana em 2015.

$\mathrm{QQ}_{08}$ Preferências de ensino.

$\mathrm{QQ}_{09}$ Qual (is) ferramenta(s) auxiliar(es) você utiliza em suas aulas?

$\mathrm{QQ}_{10}$. Qual é o melhor método de avaliar o conhecimento?

$\mathrm{QQ}_{11}$. As principais dificuldades enfrentadas na sala de aula no que diz respeito ao ensino.

Fonte: Elaborado pelos autores.

O questionário aplicado nos docentes foi baseado no estudo elaborado por Valente, Abib e Kusnik (2007). Referente aos quatro estilos de aprendizagem tem-se o quadro 3 que os descreve segundo Kolb (1984).

O Inventário de David Kolb é composto por 9 sentenças e estas são compostas por 4 frases. Cada frase recebe um peso dentro da sentença, em que o respondente deve classificar de 1 até 4 cada uma das frases de acordo a preferência, comportamento e atitude, auto avaliando suas ações no momento da aprendizagem (Schmitt e Domingues, 2016). A classificação 1 indica a frase com menor nível de identificação e a 4 com maior grau de identificação. No quadro 4 é exposto o Inventário de David Kolb, este instrumento foi respondido tanto pelos discentes, quanto pelos docentes (utilizando escala de concordância de 1 a 4). 
Quadro 3. Estilos de aprendizagem

\begin{tabular}{|c|c|}
\hline ACOMODADOR [EC - EA $]$ & ASSIMILADOR [CA - OR] \\
\hline $\begin{array}{l}\text { É denominado acomodador porque procura } \\
\text { adaptar o aprendido para seu próprio uso, usando } \\
\text { a criatividade para mudar e fazer melhor. Gosta de } \\
\text { estar comssoas, mas por vezes é tido como } \\
\text { impaciente e, de certo modo, "pressionador". É } \\
\text { mais comum utilizarem o instinto interno no lugar } \\
\text { da análise lógica. }\end{array}$ & $\begin{array}{l}\text { Pessoas com esse estilo procuram analisar, } \\
\text { organizar e assimilar partes da informação, } \\
\text { transformando-as em um todo integrado. } \\
\text { Sobressai-se no raciocínio indutivo, tem } \\
\text { facilidade para criar modelos teóricos e } \\
\text { prefere trabalhar sozinho. São mais focados } \\
\text { em ideias e conceitos abstratos. }\end{array}$ \\
\hline CONVERGENTE [CA - EA] & DIVERGENTE $[\mathrm{EC}-\mathrm{OR}]$ \\
\hline $\begin{array}{l}\text { Tende a convergir ou a tomar decisões } \\
\text { rapidamente, procura por uma resposta correta e } \\
\text { chega ao essencial com muita rapidez. Integra } \\
\text { teoria e prática: testa as informações, experimenta } \\
\text { coisas, vê como funcionam e aprende fazendo. } \\
\text { Prefere trabalhar sozinho e lidar com objetos em } \\
\text { vez de pessoas. São mais atraídos por tarefas } \\
\text { técnicas e problemas ao invés de questões } \\
\text { interpessoais ou sociais. }\end{array}$ & $\begin{array}{l}\text { É chamado divergente porque pode ver as } \\
\text { coisas de diferentes perspectivas e combinar } \\
\text { relacionamentos em um todo significativo. } \\
\text { Gosta de trabalhar em grupos, possui } \\
\text { facilidade para gerar ideias, propor } \\
\text { alternativas, reconhecer problemas, ver } \\
\text { situações concretas a partir de várias } \\
\text { perspectivas e de compreender as pessoas. }\end{array}$ \\
\hline
\end{tabular}

Fonte: Elaborado pelos autores adaptado de Kolb (1984).

Além dos dados coletados por meio dos questionários foi solicitado junto a coordenação do curso os CRAS e as notas do ENEM utilizadas pelos discentes no ingresso à instituição, a fim de realizar testes estatísticos para identificar se havia diferenças no rendimento acadêmico conforme a nota do ENEM.

Com relação ao rendimento acadêmico, Leite Filho e outros (2008) expõem que as IES prédeterminam critérios e formas de avaliações baseadas no perfil dos acadêmicos que desejam formar, de modo que, esses critérios e formas devem servir como base para mensurar e julgar suas competências. Luckesi (2002) cita que uma das questões mais problemáticas no ensino é a identificação da forma de reconhecimento do rendimento acadêmico, pois cada IES pode determinar os critérios que compõe o CRA, bem como a sua fórmula de cálculo. Nesta pesquisa utilizou-se o método da IES dos estudantes que representam a amostra do estudo e que está representado na equação 1:

$$
C R=\frac{\sum(N F \times C H)}{10 \times \sum C H}
$$

$\mathrm{CR}=$ coeficiente de rendimento

$\mathrm{NF}=$ nota final na disciplina/unidade curricular, expressa de 0,0 a 10,0

$\mathrm{CH}=$ carga horária total da disciplina/unidade curricular

As respostas dos questionários foram tabuladas em planilha eletrônica, sendo agrupadas pelo Registro Acadêmico (RA), informações que foram relacionadas com o CRA e a nota do ENEM, para posteriormente serem utilizadas na análise dos resultados.

Na etapa correspondente à análise dos dados foi necessária a utilização de alguns métodos estatísticos. Referente aos testes de diferenças de médias, utilizou-se a categoria de testes não paramétricos, a escolha deste tipo de testes justifica-se por haver variáveis de natureza nominal e ordinal. 
Quadro 4. Inventário de identificação do estilo de aprendizagem

O teste a seguir visa à identificação do seu estilo de aprendizagem a partir dos aspectos que mais valoriza no seu processo de aprendizagem. Assim, são apresentados 9 conjuntos de 4 frases.

Ordene as frases de cada conjunto na escala de intensidade de 1 a 4, sendo:

1. PIOR maneira que julga caracterizar (representar) sua maneira de aprender.

4. MELHOR maneira que julga caracterizar (representar) sua maneira de aprender

\begin{tabular}{|c|c|c|c|}
\hline Escolho: __ & Experimento: __ & Envolvo-me: __ & Sou prático: _ \\
\hline Sou receptivo: _ & $\begin{array}{l}\text { Esforço-me por ser } \\
\text { coerente: }\end{array}$ & Analiso: _ & Sou imparcial: __ \\
\hline Sinto: _ & Observo: _ & Penso: _ & Ajo: _ \\
\hline Aceito a situação: __ & Corro riscos: __ & Avalio a situação: _ & Presto atenção: __ \\
\hline $\begin{array}{l}\text { Utilizo a minha } \\
\text { intuição: }\end{array}$ & Obtenho resultados: & Utilizo a lógica: __ & Questiono: _ \\
\hline Prefiro a abstração: _ & $\begin{array}{l}\text { Prefiro a } \\
\text { observação: }\end{array}$ & $\begin{array}{l}\text { Prefiro as coisas } \\
\text { concretas: }\end{array}$ & Prefiro a ação: __ \\
\hline Vivo o presente: _ & Reflito: _ & Projeto-me no futuro: & Sou pragmático: _ \\
\hline $\begin{array}{l}\text { Apoio-me na minha } \\
\text { experiência: }\end{array}$ & Observo: _ & Conceitualizo: __ & Experimento: _ \\
\hline Concentro-me: _ & Sou reservado: _ & Racionalizo: __ & Responsabilizo-me: \\
\hline
\end{tabular}

Fonte: Elaborado pelos autores adaptado de Kolb (1984).

O teste de Kruskal-Wallis foi utilizado quando haviam mais de dois grupos amostrais e Mann-Whitney na comparação de dois grupos amostrais ou como teste post hoc do Kruskal-Wallis. Cabe destacar que o parâmetro do nível de confiança foi de $95 \%$ (p-valor $<0,05)$. Para a verificação das relações inferenciais empregou-se a técnica de regressão linear simples.

\section{Caracterização da amostra}

Com o intuito de identificar as características dos discentes foram solicitadas algumas informações referentes aos seus dados pessoais e às preferências acadêmicas, os quais possibilitaram a realização de uma análise estatística descritiva. O quadro 5 expõe a caracterização dos respondentes discentes.

A maior parte dos respondentes são do primeiro ano, do gênero feminino $(55,32 \%)$ e que possui média de idade menor que a dos homens. A maioria dos respondentes cursou o Ensino Médio em escolas públicas, sendo que a área do conhecimento que os alunos possuem maior média de facilidade auto atribuída é a interpretação de texto. Com as respostas obtidas dos docentes foi possível construir o quadro 6 a fim de identificar o perfil dos respondentes.

Cada graduação docente foi considerada de forma individual. Observa-se que a maioria dos professores é do gênero feminino, sendo que 47,83\% possuem mestrado, com diversificado tempo de experiência. Quanto aos estilos de aprendizagem dos discentes e docentes obtiveram-se os resultados demonstrados no quadro 7 .

Com um perfil que integra a prática e a teoria, o estilo convergente somente não possuía maioria absoluta na turma do $2^{\circ}$ ano, o qual compartilhava o mesmo número de respondentes com o estilo assimilador, nota-se que $45,74 \%$ dos discentes e $34,78 \%$ dos docentes caracterizam-se neste estilo. Os assimiladores, que em geral são indivíduos que 
têm facilidade com modelos teóricos e mais focados em ideias, representaram 36,17\% dos alunos e $43,48 \%$ dos professores do curso. Este resultado dos discentes pode advir do perfil prático do curso de Ciências Contábeis e embasado pelos conceitos teóricos de Kolb (1984), pois segundo o autor os convergentes são mais atraídos por tarefas técnicas e resolução de problemas, ou seja, são menos preocupados com discussões interpessoais.

Quadro 5. Caracterização da amostra do discentes

\begin{tabular}{|c|c|c|c|}
\hline CARACTERÍSTICAS & RESULTADOS & CARACTERÍSTICAS & RESULTADOS \\
\hline \multirow{4}{*}{ Ano cursado } & $1^{\circ}$ ano: $28,72 \%$ & \multirow{4}{*}{$\begin{array}{l}\text { Já reprovou em } \\
\text { alguma disciplina }\end{array}$} & \multirow{4}{*}{$\begin{array}{c}13,83 \% \text { dos } \\
\text { discentes }\end{array}$} \\
\hline & $2^{\circ}$ ano: $26,60 \%$ & & \\
\hline & $3^{\circ}$ ano: $22,34 \%$ & & \\
\hline & $4^{\circ}$ ano: $22,34 \%$ & & \\
\hline \multirow[b]{2}{*}{ Gênero } & $55,32 \%$ feminino & \multirow{2}{*}{$\begin{array}{l}\text { Melhor método de } \\
\text { avaliar o } \\
\text { conhecimento }\end{array}$} & \multirow{2}{*}{$\begin{array}{c}\text { Avaliação } \\
\text { descritiva } \\
(52,13 \%) \\
\end{array}$} \\
\hline & $44,68 \%$ masculino & & \\
\hline \multirow{2}{*}{ Média de idade } & 22,2 feminino & \multirow{2}{*}{$\begin{array}{c}\text { Facilidade em } \\
\text { matemática }\end{array}$} & \multirow{2}{*}{$\begin{array}{l}\text { Média geral: } \\
7,31\end{array}$} \\
\hline & 22,9 masculino & & \\
\hline \multirow{3}{*}{$\begin{array}{l}\text { Modalidade Ensino } \\
\text { Médio }\end{array}$} & $79,78 \%$ escolas públicas & \multirow{3}{*}{$\begin{array}{l}\text { Facilidade em } \\
\text { interpretação de } \\
\text { texto }\end{array}$} & \multirow{3}{*}{$\begin{array}{l}\text { Média geral: } \\
7,34\end{array}$} \\
\hline & $7,45 \%$ escolas particulares & & \\
\hline & $12,77 \%$ mista & & \\
\hline $\begin{array}{l}\text { Possuir outra } \\
\text { graduação }\end{array}$ & $14,89 \%$ dos respondentes & $\begin{array}{l}\text { Facilidade em } \\
\text { raciocínio lógico }\end{array}$ & $\begin{array}{l}\text { Média geral: } \\
7,18\end{array}$ \\
\hline
\end{tabular}

Fonte: Elaborado pelos autores.

Quadro 6. Caracterização da amostra de docentes

\begin{tabular}{|c|c|c|c|}
\hline CARACTERÍSTICAS & RESULTADOS & CARACTERÍSTICAS & RESULTADOS \\
\hline Gênero & $\begin{array}{l}52,17 \% \text { feminino } \\
47,83 \% \text { masculino }\end{array}$ & Maior titulação & $\begin{array}{l}\text { Graduação: 4,35\% } \\
\text { Especialização: } 17,39 \% \\
\text { Mestrado: } 47,83 \% \\
\text { Doutorado: } 30,43 \%\end{array}$ \\
\hline Formação & $\begin{array}{l}\text { Administração: } 5 \\
\text { Ciências Contábeis: } 10 \\
\text { Direito: } 2 \text {; Ed. Física: } 1 \\
\text { Filosofia: } 2 \text {; Letras: } 1 \\
\text { Matemática: } 2\end{array}$ & $\begin{array}{l}\text { Tempo de } \\
\text { experiência }\end{array}$ & $\begin{array}{l}\text { O a } 5 \text { anos: } 26,09 \% \\
6 \text { a } 13 \text { anos: } 26,09 \% \\
14 \text { a } 22 \text { anos: } 26,09 \% \\
23 \text { a } 32 \text { anos: } 21,73 \%\end{array}$ \\
\hline
\end{tabular}

Fonte: Elaborado pelos autores.

Quadro 7. Estilos de Aprendizagem docente e discente

\begin{tabular}{lccccccc|cc}
\hline & \multicolumn{7}{c|}{ DISCENTES } & \multicolumn{2}{c}{ DocENTES } \\
\cline { 2 - 10 } & $\mathbf{1}^{\mathbf{0}}$ Ano & $\mathbf{2}^{\mathbf{0}}$ Ano & $\mathbf{3}^{\mathbf{0}}$ Ano & $\mathbf{4}^{\mathbf{0}}$ Ano & Totais & $\mathbf{\%}$ & \multicolumn{2}{c}{ Totais } & $\%$ \\
\hline Acomodador & 1 & 3 & 3 & 3 & 10 & 10,64 & 3 & 13,04 \\
Assimilador & 11 & 9 & 7 & 7 & 34 & 36,17 & 10 & 43,48 \\
Convergente & 14 & 9 & 10 & 10 & 43 & 45,74 & 8 & 34,78 \\
Divergente & 1 & 4 & 1 & 1 & 7 & 7,45 & 2 & 8,70 \\
\hline Totais & 27 & 25 & 21 & 21 & 94 & - & 23 & - \\
\hline$\%$ & 28,72 & 26,60 & 22,34 & 22,34 & - & 100 & - & 100 \\
\hline
\end{tabular}

Fonte: Elaborado pelos autores. 
Os acomodadores, por sua vez, têm como característica adaptar o aprendido para uso próprio, ou seja, espelham-se na análise de soluções propostas por terceiros, aplicam a sua realidade (Kolb, 1984) e possuem facilidade em serem criativos, estes representaram a terceira maior faixa dentre os discentes, com 10,64\% e 13,04\% dos docentes. Por último, apareceram os divergentes com 7,45\% dos alunos e $8,70 \%$ dos professores, tal estilo caracteriza-se pelas diferentes percepções que possuem perante uma situação, além da sua facilidade de trabalhar em grupo, pois são interessados nas relações interpessoais, com tendência a serem imaginativos e emocionais (Kolb, 1984).

\section{Resultados}

A análise das diferenças estatísticas foram realizadas a partir de três agrupamentos distintos: (i) Rendimento Acadêmico: identificar diferenças no rendimento acadêmico de acordo com características dos discentes; (ii) Estilo de Aprendizagem: verificar se há diferenças no estilo de aprendizagem conforme características ou rendimento acadêmico dos respondentes; e (iii) Relação Discente x Docente: constatar se o desempenho acadêmico e algumas características hipotetizadas são distintas entre os grupos analisados. Foram elencados 26 possíveis agrupamentos (quadro 8), os quais foram submetidos aos testes de diferenças de média, cujo aqueles que apontaram significância estatística são destacados e detalhados individualmente com seus respectivos cotejamentos com a literatura.

Dos vinte e seis testes de diferença de média realizados, sete apresentaram significância estatística, conforme destacado no quadro 8, sendo que as "horas semanais dedicadas aos estudos extraclasses" foram diferentes para dois agrupamentos: Rendimento Acadêmico e Estilos de Aprendizagem dos Discentes. Com isso, os alunos que dedicam mais tempo ao estudo extraclasse tendem a um rendimento acadêmico acima da média, além de que há diferenças estatísticas nas horas dedicadas aos estudos entre os indivíduos de diferentes estilos de aprendizagem.

Ainda com relação o quadro 8 , as demais características verificadas apresentaram diferenças em somente um dos grupos analisados. O CRA apresentou-se distinto ao ser comparado entre o rendimento acadêmico dos discentes que reprovaram em pelo menos alguma disciplina com os demais estudantes (quadro 9).

Nota-se que 13,83\% dos discentes já reprovaram em pelo menos uma disciplina do curso, a média de rendimento acadêmico do grupo dos reprovados foi de 0,6909, apresentando diferença de 0,1358 em relação ao grupo dos não reprovados. O teste de Mann-Whitney comprova estatisticamente que os alunos reprovados possuem coeficiente menor mesmo desconsiderando a disciplina em que já houve reprovação, pois há tendência de um menor desempenho não só na matéria reprovada como também nas demais.

O resultado evidenciado remete as afirmações de Kolb (1984) ao pontuar que a aprendizagem é estabelecida por meio das percepções vivenciadas, ou seja, mesmo em disciplinas distintas o indivíduo absorve o conhecimento com a mesma percepção e processamento, com isso o desempenho geral é tendenciado.

Para realizar a análise das horas de estudos $\left(\mathrm{Q}_{12}\right)$ com o rendimento acadêmico foi necessário agrupar a amostra em dois grupos. Assim, o Grupo 1 (G1) corresponde aos estudantes que dedicavam de 0 a 3 horas aos estudos semanais (até o $2^{\circ}$ quartil) e o Grupo 2 (G2) aqueles que dedicavam de 4 a 8 horas semanais (acima do $2^{\circ}$ quartil). 
Posteriormente, foi realizada a aplicação do teste de Mann-Whitney que apontou uma significância entre as horas de estudos semanais com o desempenho acadêmico, conforme observado no quadro 10.

Quadro 8. Agrupamentos testados

\begin{tabular}{|c|c|c|c|}
\hline CORRELAÇÃo & GRUPO & CORRELAÇÃo & GRUPO \\
\hline Gênero/Idade & Rend. Acad. & Modalidade de Ensino Médio & Est. Apr. \\
\hline $\begin{array}{l}\text { Modalidade de Ensino } \\
\text { Médio } \\
\end{array}$ & Rend. Acad. & Reprovação & Est. Apr. \\
\hline $\begin{array}{l}\text { Modalidade de ingresso na } \\
\text { instituição }\end{array}$ & Rend. Acad. & Outra graduação & Est. Apr. \\
\hline $\begin{array}{l}\text { Reprovação em alguma } \\
\text { disciplina } \\
\end{array}$ & Rend. Acad. & Método de avaliação & Est. Apr. \\
\hline Outra graduação & Rend. Acad. & $\begin{array}{l}\text { Horas semanais de estudos } \\
\text { extraclasses }\end{array}$ & Est. Apr. \\
\hline Método de avaliação & Rend. Acad. & Assimilador x Acomodador & Est. Apr. \\
\hline $\begin{array}{l}\text { Horas semanais de estudos } \\
\quad \text { extraclasses }\end{array}$ & Rend. Acad. & $\begin{array}{l}\text { Facilidade em matemática, } \\
\text { interpretação de texto e } \\
\text { raciocínio lógico. }\end{array}$ & Est. Apr. \\
\hline Nota ingresso do ENEM & Rend. Acad. & $\begin{array}{l}\text { Estilos de Aprendizagem e } \\
\text { Rendimento Acadêmico }\end{array}$ & Est. Apr. \\
\hline Facilidade em matemática & Rend. Acad. & $\begin{array}{l}\text { Estilos de Aprendizagem } \\
\text { Convergente x Acomodador }\end{array}$ & Est. Apr. \\
\hline $\begin{array}{l}\text { Facilidade em } \\
\text { interpretação de texto }\end{array}$ & Rend. Acad. & $\begin{array}{l}\text { Estilos de Aprendizagem x } \\
\text { Método de avaliação }\end{array}$ & $\begin{array}{l}\text { Rel. Disct. x } \\
\text { Doct. }\end{array}$ \\
\hline $\begin{array}{l}\text { Facilidade em raciocínio } \\
\text { lógico }\end{array}$ & Rend. Acad. & $\begin{array}{l}\text { Estilos de Aprendizagem x Horas } \\
\text { lecionadas por turma }\end{array}$ & $\begin{array}{l}\text { Rel. Disct. x } \\
\text { Doct. }\end{array}$ \\
\hline Ano cursado & Est. Apr. & $\begin{array}{l}\text { Reprovações dos discentes x } \\
\text { Experiência dos Docentes }\end{array}$ & $\begin{array}{l}\text { Rel. Disct. x } \\
\text { Doct. }\end{array}$ \\
\hline Gênero/Idade & Est. Apr. & $\begin{array}{l}\text { Estilos de Aprendizagem dos } \\
\text { docentes x Reprovações }\end{array}$ & $\begin{array}{l}\text { Rel. Disct. x } \\
\text { Doct. }\end{array}$ \\
\hline
\end{tabular}

Fonte: Elaborado pelos autores.

Quadro 9. Qo6: Já reprovou em alguma matéria do curso de ciências contábeis?

\begin{tabular}{|c|c|c|c|c|c|c|}
\hline DESCRIÇÃO & $\overline{\mathbf{X}}$ & SD & ME & RESPOSTAS VÁLIDAS & $\%$ & $\begin{array}{c}\text { MANN- } \\
\text { WHITNEY }\end{array}$ \\
\hline Sim & 0,6909 & 0,0639 & 0,7158 & 13 & 13,83 & $\neq$ \\
\hline Não & 0,8267 & 0,0649 & 0,8335 & 81 & 86,17 & $\neq$ \\
\hline
\end{tabular}

Fonte: Elaborado pelos autores.

Ao final dos resultados demonstrados pelo teste de Mann-Whitney é perceptível que estatisticamente os alunos que dedicam mais de 4 horas semanais aos estudos possuem maior rendimento acadêmico que os pertencentes ao $\mathrm{G} 1$. O resultado verificado diverge do estudo de Miranda e outros (2013) que apontou relação inconclusiva entre o tempo semanal de estudos e o rendimento acadêmico, mas apoia a afirmação de Silva et al. (2015) que ressaltam que a diminuição das horas de estudos extraclasse compromete o desempenho acadêmico.

Para verificar se havia uma relação entre a nota do ENEM e o CRA foi utilizada a regressão simples. A relação obtida com a aplicação do teste de regressão foi positiva $\beta=0,330$ e 
significante $\mathrm{p}$-valor $=0,002$, indicando que quanto maior a nota do aluno no ENEM, maior tende a ser o CRA do discente. Os demais indicadores podem ser observados no quadro 11.

Quadro 10. $Q_{12:}$ Quanto tempo semanalmente você dedica aos estudos além das horas aulas?

\begin{tabular}{lccccccc}
\hline OPÇÕES & DESCRIÇÃo & $\overline{\mathbf{X}}$ & SD & ME & $\begin{array}{c}\text { RESPOSTAS } \\
\text { VÁLIDAS }\end{array}$ & $\%$ & $\begin{array}{c}\text { MANN- } \\
\text { WHITNEY }\end{array}$ \\
\hline G1 & O-3 horas & 0,7930 & 0,0822 & 0,8109 & 62 & 65,96 & $\neq$ \\
G2 & +4 horas & 0,8367 & 0,0670 & 0,8552 & 32 & 34,04 & $\neq$ \\
\hline
\end{tabular}

Fonte: Elaborado pelos autores.

Quadro 11. Regressão simples ENEM x CRA

$\mathrm{R}^{2}=10,90 \%$

$\mathrm{R}^{2}$ ajustado $=9,80 \% \quad$ Durbin-Watson $=1,953$

Regressão $=0,054 \quad \mathrm{~F}=10,392$

\begin{tabular}{ll} 
Resíduo $=0,443 \quad$ Sig. $=0,002$ \\
\hline
\end{tabular}

Total $=0,497$

Fonte: Elaborado pelos autores.

Com o objetivo de verificar se alunos com maiores notas no ENEM possuem desempenho acadêmico distinto dos com menores notas dividiu-se a amostra em quartis, sendo agrupados em G1 que corresponde a duas partes inferiores do $2^{\circ}$ quartil, e G2 correspondente às partes superiores ao $2^{\circ}$ quartil, sequencialmente foi aplicado o teste de Mann-Whitney, conforme o quadro 12.

Quadro 12. Análise das notas de ingresso do ENEM

\begin{tabular}{lccccccc}
\hline Grupo & Nota & $\overline{\mathbf{X}}$ & SD & ME & $\begin{array}{c}\text { ResPostAS } \\
\text { VÁlidAS }\end{array}$ & $\%$ & $\begin{array}{c}\text { MANN- } \\
\text { WHitNEY }\end{array}$ \\
\hline G1 & 599,35 a 618,90 & 0,7949 & 0,0822 & 0,8107 & 51 & 54,3 & $\neq$ \\
G2 & 621,00 a 714,00 & 0,8233 & 0,0748 & 0,8489 & 43 & 45,7 & $\neq$ \\
\hline
\end{tabular}

Fonte: Elaborado pelos autores.

Ao analisar o quadro 12 constata-se que há diferenças significantes no desempenho acadêmico dos discentes com maior nota no ENEM. Esses achados indicam a importância de observar o desenvolvimento do discente nas fases anteriores ao Ensino Superior, visto que quanto maior a nota no ENEM, que é uma forma de avaliar o conhecimento desenvolvimento no Ensino Médio, melhor tende a ser os resultados na graduação de nível superior.

Para verificar as diferenças inerentes a $Q_{13}$ (Facilidade em Matemática), as respostas obtidas foram divididas em quartis e agrupadas por G1 que correspondeu aos respondentes classificados até o $2^{\circ}$ quartil, e o G2 que englobou as respostas acima do $2^{\circ}$ quartil (quadro 13). Com a aplicação do teste de Mann-Whitney foi identificado que os postos de média no G2 são maiores que o G1, ou seja, alunos com maior facilidade em Matemática possuem melhor rendimento acadêmico.

Cabe destacar que a facilidade neste componente curricular é aquele auto atribuída pelos estudantes e representa uma variável não explorada até então e que pode ser confirmada ou contraposta em estudos futuros. A identificação e habilidade em lidar com problemas matemáticos apresenta-se como propulsores do rendimento acadêmico nos discentes de 
Ciências Contábeis abordados neste trabalho, isso pode ser atribuído em partes ao perfil do curso que exige a utilização desse tipo de conhecimento.

Quadro 13. $Q_{13:}$ Facilidade em matemática

\begin{tabular}{lccccccc}
\hline GruPo & RESPOSTAS & $\overline{\mathbf{X}}$ & SD & ME & $\begin{array}{c}\text { RESPOSTAS } \\
\text { VÁLIDAS }\end{array}$ & \% & $\begin{array}{c}\text { MANN- } \\
\text { WHITNEY }\end{array}$ \\
\hline G1 & 2 a 8 & 0,7939 & 0,0826 & 0,8035 & 70 & 74,47 & $\neq$ \\
G2 & 8,1 a 10 & 0,8487 & 0,0541 & 0,8588 & 24 & 25,53 & $\neq$ \\
\hline
\end{tabular}

Fonte: Elaborado pelos autores.

Logo, podem ser promovidos grupos de estudos e disciplinas no decorrer da graduação que auxiliem o estudante a maximizar suas habilidades em Matemática, e possivelmente alcançar melhores resultados nas demais matérias estudadas. Outro fator que mereceu atenção foram as horas dedicadas aos estudos em períodos externos aos de aula, sendo possível analisar o tempo dedicado aos estudos extraclasse entre os estilos de aprendizagem. Foi identificado que mais de $3 / 5$ dos alunos dedicam de uma a três horas semanais aos estudos.

Na sequência, ao aplicar o teste de Kruskal-Wallis foi verificado que há diferenças estatísticas entre as horas dedicadas por um ou mais estilos, se comparados aos outros. A fim de identificar entre quais estilos havia essas divergências foi aplicado o teste post hoc de Mann-Whitney, obtendo-se os dados apresentados no quadro 14.

Quadro 14. Teste Mann-Whitney: Horas dedicadas aos estudos entre os estilos

\begin{tabular}{|c|c|c|c|c|c|c|}
\hline EstILOS & O-3 HORAS & + 4 HORAS & ACOMOD. & Assimil. & Converg. & DIVERG. \\
\hline Acomodador & $100 \%$ & $0 \%$ & - & $\neq$ & $=$ & $=$ \\
\hline Assimilador & $52,94 \%$ & $47,06 \%$ & $\neq$ & - & $=$ & $=$ \\
\hline Convergente & $67,44 \%$ & $32,56 \%$ & $=$ & $=$ & - & $=$ \\
\hline Divergente & $71,43 \%$ & $28,57 \%$ & $=$ & $=$ & $=$ & - \\
\hline
\end{tabular}

Fonte: Elaborado pelos autores.

Ao aplicar o teste de Mann-Whitney foi identificada diferença estatística de horas dedicadas aos estudos entre os grupos acomodador e assimilador. Ao verificar as médias de horas percebe-se que $100 \%$ dos acomodadores dedicam de 0 a 3 horas semanais aos estudos, enquanto nos assimiladores este percentual foi de 52,94\%. A base teórica elaborada por Kolb (1984) expõe que os acomodadores agem por instinto ou baseados em informações obtidas de terceiros, além de possuírem uma tendência de aversão a análise lógica. Já os assimiladores tendem a explorar os modelos analíticos e refletir a lógica e valores práticos das teorias. Esses conceitos podem justificar o resultado desta análise quando, ceteris paribus, não são considerados fatores sociais que impedem maior dedicação aos estudos extraclasse, como dedicação profissional, entre outros.

Após efetuar os testes entre o rendimento acadêmico com as características discentes, foi realizada uma análise do rendimento acadêmico com os estilos de aprendizagem. Com o objetivo de verificar entre quais estilos havia diferenças foi realizado o teste post hoc de Mann-Whitney, os resultados obtidos são mostrados no quadro 15. Os testes estatísticos aplicados apontaram diferenças entre os CRAS dos acomodadores com os convergentes, cabe destacar que, estes são os grupos com a menor e a maior média de coeficiente acadêmico, respectivamente. 
Os resultados são condizentes com os apresentados por Basílio e Vasconcellos (2011), nos quais os estilos convergente e assimilador obtiveram melhor desempenho acadêmico, porém Leite Filho e outros (2008) verificaram em seus estudos maior desempenho nos alunos com estilo de aprendizagem divergente. Além disso, Leite Filho e outros (2008) não encontraram diferenças no desempenho acadêmico entre os estilos de aprendizagem, contudo os autores destacam que os resultados podem variar de acordo com a amostra e o ambiente na qual ela está inserida.

Quadro 15. Teste de Mann-Whitney: Significância estatística entre os estilos de aprendizagem

\begin{tabular}{lccccc}
\hline Estilos & $\overline{\mathbf{X}}$ CRA & ACOMOD. & AssimiL. & CONVERG. & DivERG. \\
\hline Acomodador & 0,7550 & - & $=$ & $\neq$ & $=$ \\
Assimilador & 0,8146 & $=$ & - & $=$ & $=$ \\
Convergente & 0,8226 & $\neq$ & $=$ & - & $=$ \\
Divergente & 0,7040 & $=$ & $=$ & $=$ & - \\
\hline
\end{tabular}

Fonte: Elaborado pelos autores.

Cabe destacar que os resultados elencados neste teste condizem com a plataforma teórica de Kolb (1984), pois os estilos convergente e assimilador são os que possuem características mais alinhadas com o perfil da amostra, alunos do curso de Ciências Contábeis, com isso há uma maior facilidade na absorção do conhecimento.

Após as verificações buscou-se encontrar possíveis diferenças do desempenho acadêmico conforme as características dos docentes. Nessa conjuntura, foi analisado o índice de reprovações discentes por estilos de aprendizagem dos docentes, ou seja, houve a distribuição do percentual de alunos reprovados em relação aos estilos dos professores. Perante os resultados é percebido que, mesmo os assimiladores sendo maioria entre os professores, o estilo convergente é o que mais possui alunos reprovados (quadro 16), fato que pode ser justificado pelas disciplinas lecionadas por estes professores serem mais complexas e exigirem maior dedicação extraclasse por parte dos alunos.

Quadro 16. Estilos de aprendizagem dos docentes x reprovações (Disciplinas)

\begin{tabular}{lcc}
\hline ESTILOS & REPROVAÇõES & \% REPROVAÇõES \\
\hline Acomodador & - & - \\
Assimilador & 5 & 25 \\
Convergente & 14 & 70 \\
Divergente & 1 & 5 \\
\hline Totais & 20 & 100 \\
\hline
\end{tabular}

Fonte: Elaborado pelos autores.

Para obter a quantidade de reprovações foram consideradas somente aquelas em que os professores ainda lecionavam no curso em 2015, com isto $70 \%$ das reprovações advêm de disciplinas em que os professores são convergentes, é notória esta proporção, pois 34,78\% dos professores são deste estilo. Ao verificar os percentuais de reprovações por alunos, percebe-se que somente $5,88 \%$ dos assimiladores reprovaram em alguma matéria, frente a $14,29 \%$ dos divergentes, $16,28 \%$ dos convergentes e 30\% dos acomodadores, essas diferenças são fortalecidas pelos resultados expostos anteriormente, estatisticamente o desempenho dos assimiladores é maior perante os acomodadores. O perfil de 
aprendizagem pode ser uma das causas desta diferença, pois os assimiladores tendem a ter maior facilidade teórica, coincidindo com o estilo do professor.

Pelo fato do maior número de professores caracterizarem-se no estilo assimilador e o menor percentual de reprovações entre os alunos pertencerem a este mesmo estilo, são comprovados os pressupostos teóricos expostos por Kolb em 1984 quando formulou sua teoria, em que afirmava que as divergências entre os estilos de aprendizagem relacionamse com o desempenho do indivíduo. A fim de minimizar tais efeitos, Bordenave e Pereira (2011) afirmam que é evidente a necessidade de criar várias técnicas que foquem na capacidade cognitiva dos que aprendem, independentemente de seu estilo de aprendizagem, possibilitando a participação ativa do aluno no processo de aprendizagem.

\section{Conclusões}

Os estilos de aprendizagem vêm ganhando mais espaço na pesquisa e ensino, e por meio deles muitos problemas podem ser minimizados ou evitados na relação do ensinar com o aprender. A partir disso, percebeu-se a necessidade de análises que abordem os variados fatores que possam direcionar a aprendizagem e o rendimento acadêmico. Desta forma, o propósito desta pesquisa foi, a partir do objetivo elencado, analisar aspectos que permeiam o rendimento acadêmico e os estilos de aprendizagem discente e docente.

Alguns fatores, quando verificados com o desempenho acadêmico apresentaram diferenças estatisticamente significantes e possibilitaram a ampliação dos resultados da literatura por meio de novas variáveis analisadas. Adicionalmente, foi possível confirmar os achados de estudos anteriores quanto a relação da nota do ENEM com o rendimento acadêmico, e as diferenças no rendimento acadêmico conforme as horas de estudos e estilos de aprendizagem.

Nota-se alinhamento dos achados às proposições teóricas de Kolb (1984), pois o estilo que dedica maior tempo de estudo extraclasse é justamente aquele que possui característica acentuada de análise lógica e detalha os conhecimentos teóricos com a aplicação prática. Posteriormente foi comparando o estilo de aprendizagem do professor com o do aluno, pois há uma tendência de o docente ensinar da maneira com que ele julga aprender melhor. Neste escopo, verificou-se que quando os estilos entre ambos são similares, o desempenho acadêmico tende a ser melhor, e com isso percebe-se nos resultados uma redução significativa nos índices de reprovação.

Perante as verificações há uma consolidação da necessidade de haver discussões constantes dos métodos e práticas de ensino para abranger todos os tipos de absorção de conhecimento que possam existir entre os alunos (Valente, Abib e Kusnik, 2007). Ao discutir essas práticas de ensino deve-se considerar o papel que o professor desempenha na adaptação do aluno ao ensino superior, visto que todas as experiências vividas pelo discente influenciam no seu modo de aprender (Kolb, 1984).

O desenvolvimento de diferentes metodologias, que perpassem conteúdos teóricos até a aplicação conceitual em simulações e estudos de caso são caminhos que podem ser adotados para o planejamento das aulas e o alcance das diferentes formas de aprendizagem. A capacitação dos professores a fim de possibilitar a compreensão das diferentes características cognitivas dos discentes também se mostra oportuna, a fim dos profissionais contábeis formados tenham um nível superior de aprendizagem, a fim de se 
tornarem profissionais mais preparados para o mercado de trabalho, especialmente na geração de informações úteis as organizações da atualidade.

Sugere-se que estudos futuros apliquem este instrumento em outras regiões do país, instituições de ensino públicas e privadas, e outros cursos de Ensino Superior, para assim possibilitar novas análises e comparações visando ao enriquecimento teórico deste campo de pesquisa. Ainda, estudos futuros também poderiam sugerir e acompanhar ações das instituições de ensino a fim de direcionarem seus docentes com metodologias de ensino ativas, para melhor alcançarem os diferentes estilos de aprendizagem de seus discentes, a fim de aumentar o nível de aprendizagem.

Cabe destacar que as análises não podem ser generalizadas devido às limitações amostrais e temporais da pesquisa, contudo enriquecem o ramo científico e abrangem novas análises não verificadas em estudos anteriores, como as diferenças nos índices de reprovações conforme o estilo de aprendizagem do docente e discente, rendimento acadêmico e facilidade em disciplina com mensuração auto atribuída. Refutam-se afirmações advindas do senso comum, por exemplo, o fato do discente possuir outra graduação colaborar para um maior rendimento acadêmico.

Frente à discussão apresentada nota-se que as carências de ensino estão presentes desde os anos inicias da Educação Básica e são levantadas por vários estudiosos, Kolb (1984), Cerqueira (2000), Kuri (2004), Moretto (2005) e Bordenave e Pereira (2011), os quais apontam a necessidade de adaptações no atual sistema de ensino. Todavia, a utilização frequente de um instrumento que identifique a forma de aprender dos alunos, mostra-se eficaz e necessária para contornar e minimizar as dificuldades existentes. Isto posto, o Inventário de Kolb é uma das opções que podem auxiliar na identificação destas adaptações, de modo que, a plataforma teórica abordada por Kolb (1984) fomenta e sustenta o debate da busca por um ensino cíclico que atenda ao máximo as necessidades de aprendizagem existentes, pois em uma sociedade que está em constante mudança é evidente que as formas de aprender também acompanham este ritmo.

\section{Referências}

Albuquerque, L. S. Nunes, H. F. R. de A., Bastista, F. F., Luz, J. R. de M. Y Carvalho, J. R. M. de. (2015, julho). Análise dos estilos de aprendizagem dos discentes do curso de ciências contábeis da UFCG a partir do inventario de Kolb. Comunicação apresentada em XII Congresso USP de Iniciação Científica em Contabilidade. São Paulo, Brasil.

Andrade, J. X. (2007). Condicionantes do desempenho dos estudantes de contabilidade: Evidências empíricas de natureza acadêmica, demográfica e econômica. Revista de Contabilidade da UFBA, 1(1), 24-43.

Araújo, E. A. T., Camargos, M. A., Camargos, M. C. S. e Dias, A. T. (2014). Desempenho acadêmico de discentes do curso de ciências contábeis: Uma análise dos seus fatores determinantes em uma IES privada. Contabilidade Vista \& Revista, 24(1), 60-83.

Basílio, V. B. y Vasconcellos, L. (201 1, outubro). Estilos de aprendizagem e desempenho acadêmico: Um estudo dos alunos de administração da FEA-USP. Comunicação apresentada em Seminários em Administração. São Paulo, Brasil.

Bordenave, J. D. e Pereira, A. M. (2011). Estratégias de ensino-aprendizagem. Petrópolis: Vozes. 
Butzke, M. A. e Alberton, A. (2017). Estilos de aprendizagem e jogos de empresa: A percepção discente sobre estratégia de ensino e ambiente de aprendizagem. REGE. Revista Gestão, 24(1), 72-84. https://doi.org/10.1016/j.rege.2016.10.003

Campbell, M. M. (2011). Motivational systems theory and the academic performance of college students. Journal of College Teaching \& Learning, 4(7), 59-71. https://doi.org/10.19030/tlc.v4i7.1561

Campbell, L., Campbell, B. e Dickinson, D. (2000). Ensino e aprendizagem por meio das inteligências múltiplas. Porto Alegre: Artes Médicas Sul.

Cerqueira, T. C. S. (2000). Estilos de aprendizagem em universitários (Tese de Doutorado). Universidade Estadual de Campinas, Brasil.

Correia, R. R. e Moura Júnior, P. C. (2017). Aprendizagem e procrastinação: Uma revisão de publicações no período 2005-2015. REICE. Revista Iberoamericana sobre Calidad, Eficacia y Cambio en Educación, 15(2), 111-128. https://doi.org/10.15366/reice2017.15.2.006

Dib, C. Z. (1994, outubro). Estrategias no formales para la innovación en educación: Concepto, importancia y esquemas de implementación. Comunicação apresentada em International Conference Science and Mathematics Education. Concepción, Chile.

Freitas, A. A. M. (2004). Acesso ao ensino superior: Estudo de caso sobre características de alunos do ensino superior privado. Revista Inter-Ação, Educação e Movimento Social da Faculdade de Educação da UFG, 29(2), 261-276.

Miranda, G. J., da Silva Lemos, K. C., de Oliveira, A. S. e Ferreira, M. A. (2015). Determinantes do desempenho acadêmico na área de negócios. Revista Meta: Avaliação, 7(20), 175-209. https://doi.org/10.22347/2175-2753v7i20.264

Honn, D. D. e Ugrin, J. C. (2012). The effects of cognitive misfit on students' accounting task performance. Accounting Education, 27(4), 979-998. https://doi.org/10.2308/iace-50258

Kolb, D. A. (1984). Experimental learning: Experience as the source of learning and development. Upper Saddle River, NJ: Prentice-Hall.

Kolb, D. A., Baker, A. C. e Jensen, P. J. (1997). Conversational learning: An experiential approach to knowledge creation. Westport, CT: Quorum.

Kuri, N. P. (2004). Tipos de personalidade e estilos de aprendizagem: Proposições para o ensino de engenharia (Tese de Doutorado). Universidade Federal de São Carlos, Brasil.

Larsen, H. H. (2004). Experiential learning as management development: Theoretical perspectives and empirical illustrations. Developing Human Resources, 6(4), 486-503. https://doi.org/10.1177/1523422304268383

Leite Filho, G. A., Batista, I. V. C., Junior, J. P. e Siqueira, R. L. (2008, fevereiro). Estilos de aprendizagem x desempenho acadêmico. Uma aplicação do teste de Kolb em acadêmicos no curso de ciências contábeis. Comunicação apresentada em Congresso USP de Iniciação Científica em Contabilidade. São Paulo, Brasil.

Lima Filho, R. N., Bezerra, E. S. e Silva, T. B. J. (2016). Estilo de aprendizagem dos alunos do curso de ciências contábeis. Revista GUAL, 9(2), 95-112. https://doi.org/10.5007/19834535.2016v9n2p95

Lima, T. B. e Silva, A. B. (2013). Difusão das perspectivas teóricas da aprendizagem na formação de administradores. REICE. Revista Iberoamericana sobre Calidad, Eficacia y Cambio en Educación, 11(3), 5-30.

Luckesi, C. C. (2002). Maneiras de avaliar a aprendizagem. Revista Pátio, 3(12), 7-11. 
Miranda, G. J., Ferreira, M. A., Lemos, K. C. S. e Pimenta, A. S. (2013). Determinantes do desempenho acadêmico na área de negócios. Revista Meta: Avaliação, 7(20), 175-209. https://doi.org/10.22347/2175-2753v7i20.264

Moretto, P. V. (2005). Prova um momento privilegiado de estudos e não um acerto de contas. Rio de Janeiro: DP\&A Editora.

Munhoz, A. M. H. (2004). Uma análise multidimensional da relação entre inteligência e desempenho acadêmico em universitários ingressantes (Tese Doutorado). Universidade Estadual de Campinas, Brasil.

Nogueira, D. R., Costa, J. M., Takamatsu, R. T. e Reis, L. G. (2012). Fatores que impactam o desempenho acadêmico: Uma análise com discentes do curso de ciências contábeis no ensino presencial. Revista de Informação Contábil, 7(3), 51-62.

Oliveira, A. J., Raffaelli, S. C. D., Colauto, R. D. e Casa Nova, S. P. (2013). Estilos de aprendizagem e estratégias ludopedagógicas: Percepções no ensino da contabilidade. Advances in Scientific and Applied Accounting, 6(2), 236-262. https://doi.org/10.14392/ASAA.2013060206

Oliveira, K. L. e Santos, A. A. A. (2005). Compreensão em leitura e avaliação da aprendizagem em universitários. Psicologia: Reflexão e Crítica, 18(1), 118-124. https://doi.org/10.1590/So10279722005000100016

Pellón, M., Nome, S. e Arán, A. (2013). Relação entre estilos de aprendizagem e rendimento acadêmico dos estudantes do quinto ano de medicina. Revista Brasileira de Oftalmologia, 72(3), 45-67. https://doi.org/10.1590/So034-72802013000300008

Pereira, M. (2005). Ensino-aprendizagem em um contexto dinâmico. O caso de planejamento de transportes (Tese de Doutorado). Universidade de São Paulo, Brasil. https://doi.org/10.11606/T.18.2005.tde-17062005-182009

Pimentel, A. (2007). A teoria da aprendizagem experiencial como alicerce de estudos sobre desenvolvimento profissional. Estudos de Psicologia, 12, 159-168. https://doi.org/10.1590/S1413-294X2007000200008

Reis, L. G., Paton, C. e Nogueira, D. R. (2012). Estilos de aprendizagem: Uma análise dos alunos do curso de ciências contábeis pelo método Kolb. Enfoque: Reflexão Contábil, 31, 53-66. https://doi.org/10.4025/enfoque.v31i1.13853

Schmitt, C. da S. e Domingues, M. J. C. (2016). Estilos de aprendizagem: Um estudo comparativo. Avaliação, 21(2), 361-386. https://doi.org/10.1590/S1414-40772016000200004

Santos, C. A., Bacinello, E. e Hein, N. (2017). Fatores discriminantes dos estilos de aprendizagem dos acadêmicos de ciências contábeis. Revista de Educação, Ciência e Cultura, 22(3), 129-146. https://doi.org/10.18316/recc.v22i3.2848

Silva, R. F. da. (2013). Fatores que influenciam o desempenho acadêmico. Dissertação de Mestrado. Instituto de Ensino e Pesquisa do São Paulo, Brasil.

Silva, V. R. da, Oliveira, K. G. de, Rogers, P. e Miranda, G. J. (2015, setembro). Comportamento e desempenho acadêmico no curso de ciências contábeis. Comunicação apresentada em Congresso ANPCONT. Associação Nacional de Programas de Pós-graduação em Ciências Contábeis. Curitiba, Brasil.

Sobral, D. T. (1992). Inventário de estilo de aprendizagem de Kolb: Características e relação com resultados de avaliação no ensino pré-clínico. Psicologia: Teoria e Pesquisa, 8(3), 293-303.

Sobral, D. T. (2005). Estilos de aprendizagem dos estudantes de medicina e suas implicações. Revista Brasileira de Educação Médica, 29(1), 5-12. 
Sonaglio, A. L. B., Godoi, C. K. e Silva, A. B. (2013). Estilos de aprendizagem experiencial e aquisição de habilidades: Um estudo com discentes de graduação em administração em instituições de ensino superior. Administração: Ensino e Pesquisa, 14, 123-159. https://doi.org/10.13058/raep.2013.v14n1.75

Souza, G. H. S., Costa, A. C. S. C., Penedo, A. S. T., Lima, N. C., Santos, P. da C. F. e Pontes Júnior, J. F. V. (2013). Estilos de aprendizagem dos alunos versus métodos de ensino dos professores do curso de administração. RACE. Revista de Administração, Contabilidade e Economia, 12, 9-44.

Turra, S., Jacomossi, F. A. e Biavatti, V. T. (2015). Análise da preferência do estilo de aprendizagem dos alunos de graduação em ciências contábeis. Revista de Contabilidade da UFBA, 9, 118 134.

Valente, N. T. Z., Abib, D. B. e Kusnik, L. F. (2007). Análise dos estilos de aprendizagem dos alunos e professores do curso de graduação em ciências contábeis de uma universidade pública do estado do paraná com a aplicação do inventário de David Kolb. Contabilidade Vista \&̊ Revista, $18(1), 51-74$.

\title{
Breve CV dos autores
}

\author{
Alison Martins Meurer \\ Professor nos cursos de Ciências Contábeis e Administração no Centro Universitário \\ Campos de Andrade, Curitiba/PR. Estudante do Programa de Pós-Graduação em \\ Contabilidade da Universidade Federal do Paraná, Curitiba/PR. Especialista em \\ Avaliação do Ensino e da Aprendizagem pela Universidade do Oeste Paulista, Presidente \\ Prudente/SP. Graduado em Ciências Contábeis pela Universidade Tecnológica Federal \\ do Paraná, Pato Branco/PR. Integrante do Laboratório de Educação e Pesquisa Contábil \\ da Universidade Federal do Paraná, Curitiba/PR. ORCID ID: https://orcid.org/Oooo- \\ o002-3704-933X. Email: alisonmmeurer@gmail.com
}

\section{Daiana Rafaela Pedersini}

Estudante da Especialização em Gestão Contábil e Financeira da Universidade Tecnológica Federal do Paraná, Pato Branco/PR. Graduada em Ciências Contábeis pela Universidade Tecnológica Federal do Paraná, Pato Branco/PR. ORCID ID: https://orcid.org/0000-0002-2897-0880 Email: daianapedersini@hotmail.com

\section{Ricardo Adriano Antonelli}

Professor do curso de Ciências Contábeis da Universidade Tecnológica Federal do Paraná , Pato Branco/PR. Doutor em Contabilidade pelo Programa de Pós-Graduação em Contabilidade da Universidade Federal do Paraná, Curitiba/PR. Mestre em Contabilidade pelo Programa de Pós-Graduação em Contabilidade da Universidade Federal do Paraná, Curitiba/PR. Graduado em Ciências Contábeis pela Universidade Tecnológica Federal do Paraná, Pato Branco/PR. Graduado em Informática pela Universidade Estadual de Ponta Grossa, Ponta Grossa/PR. Especialista em Desenvolvimento para Ambiente de Internet utilizando Orientação a Objetos, Java e Banco de Dados Universidade Tecnológica Federal do Paraná, Pato Branco/PR. Perito Judicial desde o ano de 2011 atuando em causas cíveis e bancárias. ORCID ID: https://orcid.org/0000-0001-7606-2388. Email: rantonelli@utfpr.edu.br 


\section{Simone Bernardes Voese}

Professora do curso de Ciências Contábeis da Universidade Federal do Paraná, Curitiba/PR. Pós-doutora em Administração pela Universidade Federal do Mato Grosso do Sul, Campo Grande/MS. Doutora em Engenharia da Produção pela Universidade Federal de Santa Catarina, Florianópolis/SC. Mestre em Engenharia da Produção pela Universidade Federal de Santa Catarina, Florianópolis/SC. Graduada em Ciências Contábeis pela Universidade do Vale do Itajaí, Itajaí/SC. ORCID ID: https://orcid.org/0000-0002-5555-8250.Email: simone.voese@ufpr.br 